Results A total of 58 babies were identified with 64 episodes of medical treatment for PDA with Indomethacin or Ibuprofen. Mean gestational age was 25.5 weeks and birth weight of 737.1 grams. Mean age at treatment was 26.7 weeks with pre-treatment PDA size of $1.5-6 \mathrm{~mm}$ and platelet count of $34-602 \times 10^{9} / \mathrm{L}$.

Overall 73\% (47/64) were treated with high dose and 11\% (7/64) with low dose Indomethacin. 30\% (19/64) PDA closed post treatment, $62 \%$ (40/64) remained open.

Out of 19 successful closures 7 had initial platelet counts of $>150$ (Odds Ratio 0.31, 95\% Confidence Interval) and out of 40 unsuccessful closures 26 had initial platelet counts of $>150$ (Odds Ratio $3.18,95 \%$ Confidence Interval).

Conclusions Success of PDA closure after medical treatment was not related to the platelet counts in our study group.

\section{ANTENATAL VS POSTNATAL DETECTION OF MAJOR CONGENITAL HEART DISEASE IN A LARGE DISTRICT- GENERAL HOSPITAL IN UK; A SIX-YEAR REVIEW}

doi:10.1136/archdischild-2012-302724.1133

'S Rajendran, 'A Shastri, ${ }^{2} \mathrm{~K}$ Annam. ' Colchester General Hospital, Colchester Hospital University; ${ }^{2}$ Colchester General Hospital, Colchester Hospital University Foundation Trust, Colchester, UK

Background and Aims Major congenital heart disease (CHD) is defined as CHD that needs operative or catheter based intervention in the first year of life. National institute of clinical excellence (NICE) in March 2008 recommended screening of outflow-tracts in addition to four-chamber view as part of the anomaly scan to improve CHD detection rates. We aimed to examine the clinical spectrum of antenatally and postnatally diagnosed major CHD in our institute pre- and post-introduction of NICE guideline.

Methods This is a retrospective review over six years from Jan 2006 to Dec 2011. Data was obtained from antenatal records, patient's clinical and electronic records.

Results A total of 74 babies had major CHD diagnosed out of which 37 (50\%) were diagnosed antenatally. Antenatal diagnosis pre- and post- NICE guidelines were 12/29 (41\%)and 25/45 (55\%) respectively as also termination of pregnancies with critical CHD doubled. Common postnatal presentations included cardiovascular collapse $4(11 \%)$, cyanosis $8(22 \%)$, murmurs $12(32 \%)$, heart failure $5(13.5 \%)$, faltering growth $5(13.5 \%) .4$ babies were critically ill with severe acidosis and 3 needed intubation and ventilation prior to transfer to a tertiary unit. Median age at intervention for duct dependent lesions was 10 days and other major CHD was 5 months. Conclusions Although antenatal screening methods have improved, a large proportion of babies with CHD are still undetected. Routine Pulse-oximetry screening has proved to be an effective screening adjunct in four large studies and hence should be considered as part of early neonatal examination.

\section{PROTEASE-ACTIVATED RECEPTOR (PAR)-MEDIATED CONTRACTION OF THE CHICKEN DUCTUS ARTERIOSUS}

doi:10.1136/archdischild-2012-302724.1134

K Kartal, J Meekels, R Mohammed, S van der Sterren, E Villamor. Pediatrics, Maastricht University Medical Center, Maastricht, The Netherlands

Background and Aims PARs belong to a family of $G$ protein- coupled receptors, thus mediating the cellular effects of proteinases. PAR1 and PAR2 have been shown to be involved in regulating vascular tone. Thrombin activates PAR1, whereas trypsin activates PAR1 and PAR2. Our aim was to evaluate the functional presence of PAR1 and PAR2 in the ductus arteriosus (DA).

Methods We investigated, using wire myography, the mechanical responses induced by thrombin $(0.1$ to $3 \mathrm{U} / \mathrm{mL})$, trypsin $(0.1$ to 30 $\mathrm{U} / \mathrm{mL}$ ), the PAR1-activating peptide TFLLR- $\mathrm{NH}_{2}(1$ to $100 \mu \mathrm{mol} / \mathrm{L})$ and the PAR2-activating peptide SLIGRL- $\mathrm{NH}_{2}(0.1$ to $10 \mu \mathrm{mol} / \mathrm{L})$ in DA rings from 15-, 19-, and 21-d chicken embryos.

Results Thrombin, trypsin, and TFLLR- $\mathrm{NH}_{2}$, all caused concentration-dependent contraction of the pulmonary side of chicken DA. These contractions were not observed in the aortic side of the DA, in the femoral artery or in the pulmonary artery. Thrombin-, trypsin- and TFLLR- $\mathrm{NH}_{2}$-induced contractions were endotheliumindependent but markedly impaired by the elimination of calcium from the external medium. The contraction evoked by thrombin and trypsin increased between day 15 and 19 of incubation and was not affected by oxygen tension. SLIGRL- $\mathrm{NH}_{2}(\geq 10 \mu \mathrm{mol} / \mathrm{L})$, evoked endothelium-dependent relaxation of the DA.

Conclusions PARs are functionally present in the chicken DA but not in other vascular tissues. Recent studies demonstrate that loss of platelet number or function leads to defective DA closure. We speculate that the role of platelets in DA closure might be partially mediated through the PAR-mediated vasoactive effects of thrombin.

\section{CORONARY ARTERY ANATOMY PRIOR TETRALOGY OF FALLOT SURGERY}

doi:10.1136/archdischild-2012-302724.1135

${ }^{1} \mathrm{~S}$ Mesihovic-Dinarevic, 'Z Begic, ${ }^{1} \mathrm{~A}$ Kadic, ${ }^{1} \mathrm{M}$ Halimic, ${ }^{2} \mathrm{~S}$ Pandur, ${ }^{2} \mathrm{H}$ Vila, ${ }^{2} \mathrm{E}$ Omerbasic, II Poplata. 'Peadiatric Clinic, Clinical Centre University of Sarajevo; ${ }^{2}$ Heart Centre, Clinical Centre University of Sarajevo, Sarajevo, Bosnia-Herzegovina

Intoduction Anomalous coronary artery disease is common among patients with Tetralogy of Fallot (ToF). The need to define the morfohaemodynamics of TOF is the priority in echo laboratory prior TOF surgery.

Aim To evaluated coronary artery anatomy pre and intraoperatively by imaging technique-echocardiograhy and to establish frequency of congenital coronary arteries anomalies in patients with tetralogy of Fallot, as a plan of surgical approach in these patients.

Methods Consecutive retrospective analysis of echocardiography data patients with tetralogy of Fallot and surgical protocols of total corrections of Tetralogy of Fallot was performed.

Results During period from 01.01.2010 till 01.01.2011. at Paediatric clinic CCU Sarajevo Out pts Cardiology department 3577 pts were evaluated, $70 / 1.9 \%$ /pts with CHD and $13 / 70$ with ToF (18\%), age 25 monhts (9-60), 7 girls (53.8\%). First Group had simple ToF/n-10/and Second Group had Pentalogia Fallot (n-3 pts). Anatomical correction was performed in all pts in Sarajevo by foregin an local team. Preoperativey $2 / 15.4 \%$ /pts with ToF had anmalous origin of anterior interventricular branch of RCA crossing RVOT which was as well confirmed intraoperatively. The surgical approcah was based upon the preoperativelly echo findings, so these 2 pts had RVOT-PA graft construction. All pts from the Second Group had normal anatomy of coronary arteries pre- and intraoperatively. Conclusion Non invasive transtoracic echocardiography (TTE) is very usefull tool in deliniation of coronary artery anatomy as a part of surgical approach plan in patients with TOF which defenitly could decrease the risk of surgery.

\section{MORPHOHEMODYNAMIC MONITORING OF PULMONARY ARTERY IN PREMATURE INFANTS}

doi:10.1136/archdischild-2012-302724.1136

E Hadimujic-Konjalic, S Mesihovic-Dinarevic, S Terzic. Pediatric, Clinic Center Sarajevo, Sarajevo, Bosnia-Herzegovina

Introduction Two-dimensional echocardiography is increasingly used in Neonatal intensive care departments in order to test structure and function of the hearth because a definite diagnosis in this period is very difficult if solely based on physical examination. 